\title{
Computed tomography for detecting left atrial thrombus: a meta-analysis
}

Xiaosan Wu, Congxia Wang, Chunyan Zhang, Yan Zhang, Faming Ding, Jiaojuan Yan

Department of Cardiovascular Medicine, Second Affiliated Hospital of Medical School, Xi'an Jiaotong University, Shaanxi, China

Submitted: 1 September 2012

Accepted: 13 October 2012

Arch Med Sci 2012; 8, 6: 943-951

DOI: 10.5114/aoms.2012.32400

Copyright $\odot 2012$ Termedia \& Banach

\section{Abstract}

Introduction: Thromboembolism, usually originating from the left atrium (LA) and left atrial appendage (LAA), is a major complication of atrial fibrillation and may result in transient ischemic attack and stroke. Computed tomography (CT) is a noninvasive test for detection of LA and LAA thrombus. We sought to conduct a meta-analysis to evaluate the accuracy of CT in detecting LA/LAA thrombus.

Material and methods: The PubMed, Medline, ISI Web of Knowledge and Cochrane Library databases up to June 2012 were searched for studies comparing $\mathrm{CT}$ and transesophageal echocardiography as the reference standard in detecting LA/LAA thrombus. Meta-analysis methods were used to pool sensitivity and specificity and to construct summary receiver operating characteristic (SROC) curves.

Results: A total of 9 studies with 1646 patients were included in this meta-analysis. The publication years spanned from 2007 to 2012. For CT diagnosis of LA/LAA thrombus, the mean sensitivity and specificity were $81 \%(95 \% \mathrm{Cl}: 70-90 \%)$ and $90 \%$ (95\% Cl: $88-91 \%)$, respectively. The SROC analysis showed an area under the curve of 0.93 .

Conclusions: Computed tomography shows a good diagnostic accuracy in detecting LA/LAA thrombus with high sensitivity and specificity. Thus CT should be considered the foremost noninvasive alternative to transesophageal echocardiography for detecting LA/LAA thrombus. Randomized studies at the patient level are needed to address the potential use of CT in detecting LA/LAA thrombus.

Key words: computed tomography, transesophageal echocardiography, left atrial thrombus, meta-analysis.

\section{Introduction}

Investigation of potential embolic sources is an important diagnostic step in treating patients with acute ischemic stroke or transient ischemic attack (TIA), especially when the mechanism is considered to be embolic. Cardiogenic embolism has been estimated to be the causative factor in $20-40 \%$ of all cases of stroke [1-3]. Atrial fibrillation (AF) is the most common cause of embolic sources from the heart, and AF-related strokes constitute about $60 \%$ of all cardioembolic strokes [4]. Patients with stroke and AF frequently have concomitant potential cardiac sources of embolism and are at increased risk of recurrent embolism, despite anticoagulation $[5,6]$. The detection of certain concomitant potential cardiac sources

\author{
Corresponding author: \\ Congxia Wang PhD \\ Department \\ of Cardiovascular Medicine \\ Second Affiliated Hospital \\ of Medical School \\ Xi'an Jiaotong University \\ Xi'an, Shaanxi 710004 \\ China \\ Phone: +86 2987679770 \\ E-mail:wcx0622@gmail.com
}


of embolism in patients with stroke and AF may thus help to identify a patient group with a high risk of recurrent stroke. Furthermore, preexisting atrial thrombus should be excluded before the cardioversion of atrial fibrillation or catheter ablation to avoid embolization.

Thromboembolism, usually originating from the left atrium (LA) and left atrial appendage (LAA), is a major complication of AF and may result in TIA and stroke [7]. Accordingly, the 2007 Heart Rhythm Society/European Heart Rhythm Association/European Cardiac Arrhythmia Society consensus statement recommends pre-procedural transesophageal echocardiography (TEE) in order to screen for the presence of thrombus, especially in patients with persistent AF at the time of ablation [8]. Although TEE is still considered the gold standard to exclude LA/LAA thrombus, TEE requires special skills for proper performance and interpretation. Additionally, it is a relatively invasive test, usually performed with the patient under conscious sedation $[9,10]$.

Alternatively, a potentially non-invasive and efficacious method allowing identification of LA/LAA thrombus with reliability and accuracy comparable to TEE would be of significant clinical value. Recent advances in multi-detector spiral CT (MDCT), including improvements in temporal and spatial resolution, now allow accurate and consistent imaging of cardiac structure, including LA/LAA thrombus.

Over the last decade, mainly smaller studies have been performed among patients to determine the diagnostic value of MDCT for detecting LA/LAA thrombus, compared with TEE as the reference standard. However, sensitivity and specificity of MDCT in excluding LAA thrombus were varied in prior studies. However, the ability of MDCT to detect LA/LAA thrombus has never been systematically validated because of the small sample sizes. We therefore performed a meta-analysis to explore the potential diagnostic value of CT in detecting LA/LAA thrombus.

\section{Material and methods}

\section{Literature search}

This meta-analysis was performed in accordance with the Quality of Reporting of Meta-analysis (QUOROM) consensus guidelines and according to a protocol that prespecified outcomes, search strategies, inclusion criteria, and statistical analysis [11]. We identified studies by a literature search of the PubMed, Medline, ISI Web of Knowledge and Cochrane Library databases up to June 2012 with the following key words: "left atrial thrombus", "left atrial appendage thrombus", "left atrial filling defects" or "left atrial appendage filling defects" plus "computed tomography". In addition, we review- ed the reference lists from all relevant articles to identify additional studies if necessary. All searches were conducted independently by 2 authors. The results were compared, and any questions or discrepancies were resolved through iteration and consensus.

\section{Inclusion criteria for included studies}

We included articles in any language that (i) compared MDCT with TEE as the reference standard; (ii) had a prospective design; (iii) included patients with suspected LA/LAA thrombus; (iv) involved multi-slice CT scanners ( $\geq 16$ slice); (v) clearly stated the number of true positive, false positive, false negative, and true negative results for diagnosis of LA/LAA thrombus, or they could be calculated from the article; (vi) defined LA/LAA thrombus as an intracavitary contrast filling defect with attenuation values similar to non-enhanced tissue by MDCT; and (vii) defined LA/LAA thrombus as an intracavitary echogenic mass that could be distinguished from the surrounding tissue in more than one imaging by TEE.

\section{Data extraction and quality assessment}

Data were abstracted by use of specific data collection forms by two authors and checked for accuracy. We extracted data on study population characteristics, relevant technical information about the CT and TEE approach, and detailed reference standard specifications. We assessed methodological quality by the Quality Assessment of Diagnostic Accuracy Studies (QUADAS) tool [12] and individually tailored the guidelines for scoring each item in our review as suggested [13].

\section{Data synthesis and statistical analysis}

We used meta-analysis software (MetaDisc, Version 1.4, Unit of Clinical Biostatistics, Ramón Y Cajal Hospital, Madrid, Spain; STATA, Version 11.0, StataCorp, College Station, TX, USA) for the analysis. The pooled indices of sensitivity and specificity with the corresponding 95\% confidence intervals (CI) were calculated by using weighted averages according to the sample size of each study. The pooled estimates of positive likelihood ratio, negative likelihood ratio and diagnostic odds ratio (DOR) were computed by the DerSimonian and Laird method based on a random-effects model [14, 15].

In addition, an assessment of diagnostic threshold variation among studies was undertaken using a summary receiver operating characteristic (SROC) curve. The SROC curve (and area under the curve) represents the overall performance of the test, and depicts the trade-off between sensitivity and specificity. A symmetric curve suggests that the variability in accuracy between studies is explained, in 
part, by differences in thresholds employed by the studies.

Heterogeneity in meta-analysis refers to the degree of variability in results across studies. Heterogeneity of results across trials was assessed with a standard $\chi^{2}$ test with significance set at $p<0.10$ and an $R^{2}$ statistic with significance set at $R^{2}>50 \%$. A value of $p<0.01$ was considered statistically significant [16]. Stratified (subgroup) analyses were used to identify study design and testrelated factors responsible for heterogeneity in test accuracy.

Publication bias was assessed using several methods. Visual inspection of a funnel plot for vascular-related end-points was performed. A funnel plot provides a visual representation of each study included in the meta-analysis plotted by its effect size on the horizontal axis and variance on the vertical axis. When publication bias is not present, the funnel plot resembles an inverted funnel, with less precise studies having greater variance scattered at the bottom to either side of the more precise studies. If publication bias is present in a meta-analysis, the plot does not appear as an inverted, symmetrical funnel. Egger's weighted regression method was also used to assess publication bias $(p<0.05$ was considered representative of statistically significant publication bias) [17].

\section{Results}

\section{Trial flow}

The primary search revealed 261 reports. At review, 239 reports were excluded after reading the abstract and a further 13 reports were excluded after reading the full report because they did not meet the inclusion criteria. We finally accepted 9 reports [18-26]. Figure 1 shows the selection of studies for the study.

Table I. Criteria used to assess the methodological quality of included studies according to the QUADAS tool

\begin{tabular}{|c|c|}
\hline Quality item & Positive score \\
\hline $\begin{array}{l}\text { 1. Was the spectrum of patients representative } \\
\text { of the patients who will receive the test in practice? }\end{array}$ & Only patients suspected of stroke were included. \\
\hline 2. Were selection criteria clearly described? & $\begin{array}{l}\text { It was clear how patients were selected to undergo } \\
\text { CT scan before and during or after IVUS exam. }\end{array}$ \\
\hline $\begin{array}{l}\text { 3. Is the reference standard likely to enable correct } \\
\text { classification of the target condition? }\end{array}$ & $\begin{array}{l}\text { Transesophageal echocardiography was used as the } \\
\text { reference standard. }\end{array}$ \\
\hline $\begin{array}{l}\text { 4. Is the time period between the reference standard } \\
\text { and index test short enough to be reasonably sure that } \\
\text { the target condition did not change between the two tests? }\end{array}$ & Time period between CT and TEE was short enough. \\
\hline $\begin{array}{l}\text { 5. Did the whole sample or a random selection } \\
\text { of the sample receive verification with } \\
\text { a reference standard? }\end{array}$ & $\begin{array}{l}\text { All patients or a random selection of the patients received } \\
\text { verification with TEE. }\end{array}$ \\
\hline $\begin{array}{l}\text { 6. Did patients receive the same reference standard } \\
\text { regardless of the index test result? }\end{array}$ & $\begin{array}{l}\text { All patients received the same reference standard } \\
\text { regardless of the index test result. }\end{array}$ \\
\hline $\begin{array}{l}\text { 7. Was the reference standard independent } \\
\text { of the index test? }\end{array}$ & The reference standard was independent of the index test. \\
\hline $\begin{array}{l}\text { 8. Was the execution of the index test described } \\
\text { in sufficient detail to permit replication of the test? }\end{array}$ & $\begin{array}{l}\text { The CT scan protocol (scanner type, acquisition mode, } \\
\text { reconstruction method, length of fasting before scanning, } \\
\text { and interpreter(s)) was described. }\end{array}$ \\
\hline $\begin{array}{l}\text { 9. Was the execution of the reference standard described } \\
\text { in sufficient detail to permit its replication? }\end{array}$ & $\begin{array}{l}\text { Sufficient details or citations were reported to permit } \\
\text { replication of the TEE. }\end{array}$ \\
\hline $\begin{array}{l}\text { 10. Were the index test results interpreted without } \\
\text { knowledge of the results of the reference standard? }\end{array}$ & $\begin{array}{l}\text { Interpretation of CT findings was performed without } \\
\text { knowledge of IVUS findings. }\end{array}$ \\
\hline $\begin{array}{l}\text { 11. Were the reference standard results interpreted } \\
\text { without knowledge of the results of the index test? }\end{array}$ & $\begin{array}{l}\text { Interpretation of the TEE results was performed without } \\
\text { knowledge of the CT findings. }\end{array}$ \\
\hline $\begin{array}{l}\text { 12. Were the same clinical data available when test results } \\
\text { were interpreted as are available when the test is used } \\
\text { in practice? }\end{array}$ & $\begin{array}{l}\text { Clinical data, such as age, sex, and clinical symptoms, } \\
\text { were available when CT results were interpreted. }\end{array}$ \\
\hline $\begin{array}{l}\text { 13. Were uninterpretable and/or intermediate test results } \\
\text { reported? }\end{array}$ & $\begin{array}{l}\text { All CT results, including uninterpretable and/or } \\
\text { intermediate results, were reported. }\end{array}$ \\
\hline 14. Were withdrawals from the study explained? & $\begin{array}{l}\text { It was clear what happened to all patients who withdrew } \\
\text { from the study. }\end{array}$ \\
\hline
\end{tabular}




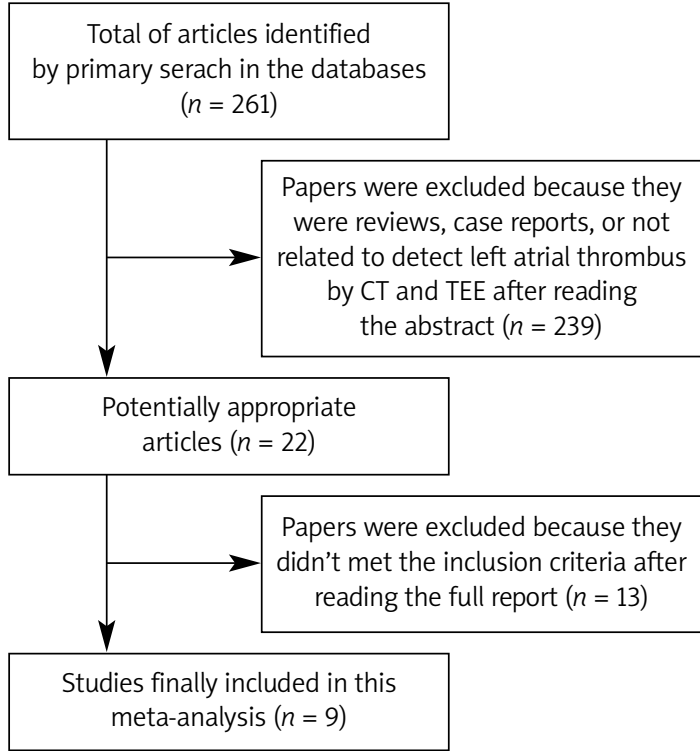

Figure 1. Trial flow diagram of the literature search resulting in 9 included studies

\section{Study characteristics}

From the literature search, 9 studies were accepted finally [18-26]. The study characteristics are shown in Table II. The publication years spanned from 2007 to 2012. Patient enrolment in single studies ranged from 51 to 402 patients. The total number of patients was 1646. All of the included reports involved patients with AF or stroke who had high risk factors for thrombus formation. All of the reports described single-center prospective cohort studies. All of the reports used contrast-enhanced CT protocols to detect LA/LAA thrombus. In order to validate the $\mathrm{CT}$ results, all the included studies required patients to undergo TEE. Of all the includ- ed studies, LA/LAA thrombus was defined as an intracavitary contrast filling defect with attenuation values similar to non-enhanced tissue by MDCT and an intracavitary echogenic mass that could be distinguished from the surrounding tissue in more than one imaging by TEE. Of the 9 included studies, 6 used a 64-slice CT scanner [18-20, 22-24], 1 used a 128-slice CT scanner [26], 2 involved 2 different types of scanners [21, 25], and 3 used a dualsource CT scanner $[19,20,26]$.

\section{Methodological quality}

Findings from the QUADAS tool for quality assessment of diagnostic accuracy studies can be found in Figure 2. Table I shows how the studies scored on each item. The quality and completeness of the reporting of studies were variable, and the studies were generally of high quality. All of the reports met the requirements for at least 8 of the items and in 8 of the 9 studies fulfilled more than 10 items of the 14-item QUADAS checklist. Ten of the QUADAS items were met by more than $75 \%$ of the studies.

\section{Quantitative data synthesis}

True-positive (TP), false-negative (FN), false-positive (FP) and true-negative (TN) results for included reports are shown in Table III. Among the 9 reports providing data on the diagnostic accuracy data of CT in detecting LA/LAA thrombus, the pooled sensitivity was $81 \%$ (95\% Cl: $70-90 \%)$ and the pooled specificity was $90 \%(95 \% \mathrm{Cl}: 88-91 \%)$ (Figure 3 ). To assess the impact of diagnostic threshold variation between studies, a symmetrical SROC curve was fitted (Figure 4). The SROC curve displays a ROC-type trade-off between sen-

Table II. Characteristics of included studies

\begin{tabular}{|c|c|c|c|c|c|c|c|c|}
\hline Study & Country & $\begin{array}{c}\text { Publication } \\
\text { year }\end{array}$ & $\begin{array}{l}\text { Number } \\
\text { of patients }\end{array}$ & $\begin{array}{l}\text { Mean age } \\
\text { [years] }\end{array}$ & $\begin{array}{l}\text { Men } \\
{[\%]}\end{array}$ & $\begin{array}{l}\text { Disease } \\
\text { status }\end{array}$ & TEE type & CT type-slice \\
\hline Dorenkamp et al. [18] & ] Germany & 2011 & 329 & 62 & 65 & $\mathrm{AF}$ & $5 \mathrm{MHz}$ & $64 \mathrm{~s}$ \\
\hline Kapa et al. [19] & USA & 2010 & 255 & 58 & 78 & $\mathrm{AF}$ & 3.5-7 MHz & $\begin{array}{c}\text { Dual-source } \\
\quad 64 \mathrm{~s}\end{array}$ \\
\hline Hur et al. [20] & South Korea & 2012 & 63 & 64 & 78 & $\mathrm{AF}$ & $5 \mathrm{MHz}$ & $\begin{array}{c}\text { Dual-source } \\
\quad 64 \mathrm{~s}\end{array}$ \\
\hline Kim et al. [21] & New York & 2007 & 223 & 57 & 82 & $\mathrm{AF}$ & 5-7 MHz & 16 and $64 \mathrm{~s}$ \\
\hline Martinez et al. [22] & USA & 2009 & 402 & 56 & 76 & $\mathrm{AF}$ & 3.5-7 MHz & $64 \mathrm{~s}$ \\
\hline Singh et al. [23] & USA & 2009 & 51 & 64 & 73 & $\mathrm{AF}$ & $7 \mathrm{MHz}$ & $64 \mathrm{~s}$ \\
\hline Tang et al. [24] & China & 2008 & 170 & 56 & 72 & $\mathrm{AF}$ & $5 \mathrm{MHz}$ & $64 \mathrm{~s}$ \\
\hline Sawit et al. [25] & New York & 2012 & 70 & 58 & 73 & $\mathrm{AF}$ & NA & $\begin{array}{c}64,128 \\
\text { and } 256 \mathrm{~s}\end{array}$ \\
\hline Hur et al. [26] & South Korea & 2011 & 83 & 63 & 67 & $\begin{array}{l}\text { Recent } \\
\text { stroke }\end{array}$ & $5-7 \mathrm{MHz}$ & $\begin{array}{c}\text { Dual-source } \\
128 \mathrm{~s}\end{array}$ \\
\hline
\end{tabular}

NA - data not available, AF - atrial fibrillation, TEE - transesophageal echocardiography 


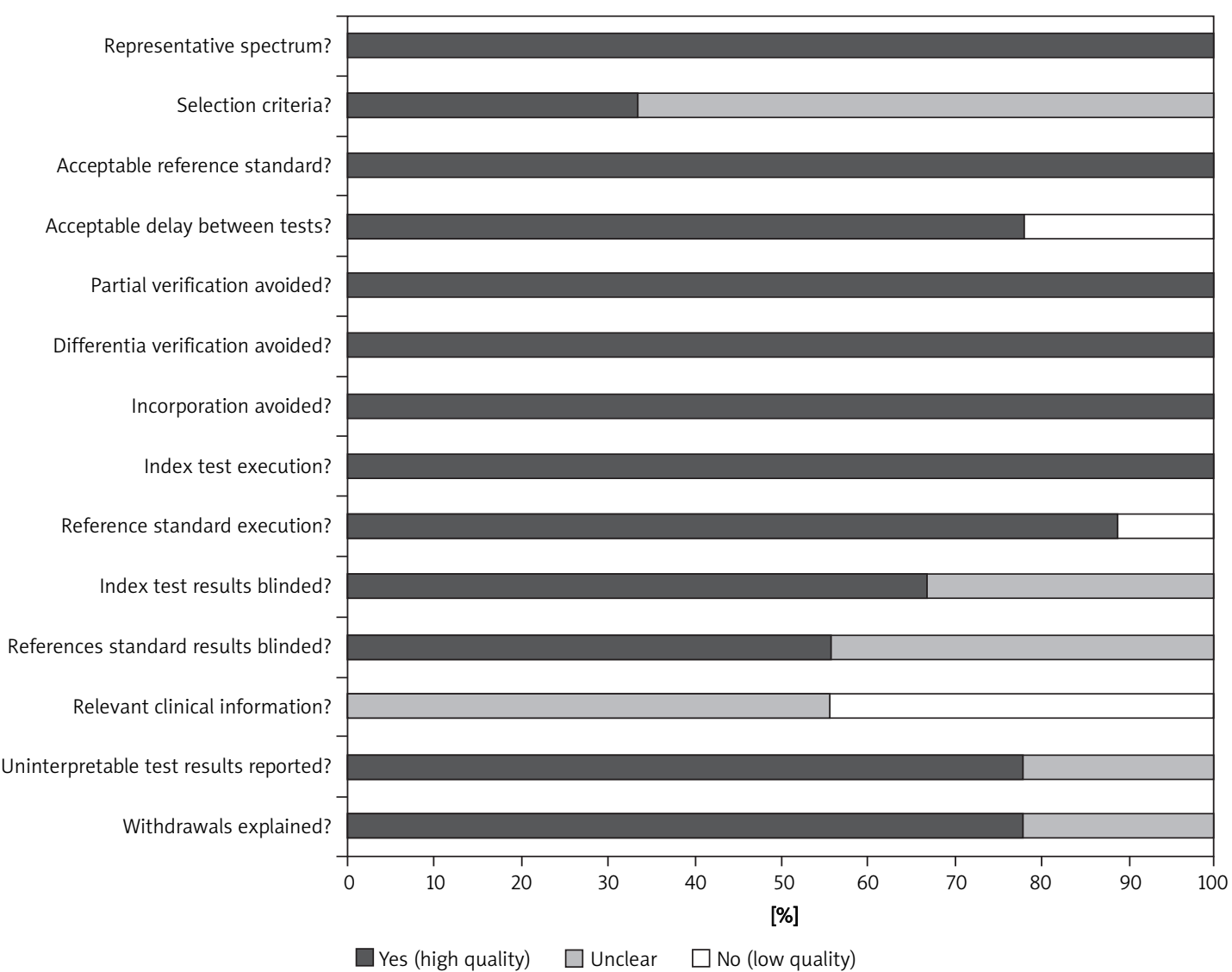

Figure 2. Methodological quality of included studies according to the QUADAS tool

Table III. Study results

\begin{tabular}{|lccccccc|}
\hline Study & N & TP & FP & FN & TN & Sensitivity (95\% Cl) & Specificity (95\% Cl) \\
\hline Dorenkamp et al. [18] & 329 & 2 & 8 & 5 & 314 & $0.29(0.04-0.71)$ & $0.98(0.95-0.99)$ \\
\hline Kapa et al. [19] & 255 & 4 & 29 & 0 & 222 & $1.00(0.40-1.00)$ & $0.88(0.84-0.92)$ \\
\hline Hur et al. [20] & 63 & 13 & 18 & 0 & 32 & $1.00(0.75-1.00)$ & $0.64(0.49-0.77)$ \\
\hline Kim et al. [21] & 223 & 3 & 42 & 0 & 178 & $1.000 .29-1.00)$ & $0.81(0.75-0.86)$ \\
\hline Martinez et al. [22] & 402 & 9 & 31 & 0 & 362 & $1.00(0.66-1.00)$ & $0.92(0.89-0.95)$ \\
\hline Singh et al. [23] & 51 & 2 & 2 & 0 & 47 & $1.00(0.16-1.00)$ & $0.96(0.86-1.00)$ \\
\hline Tang et al. [24] & 170 & 4 & 10 & 7 & 149 & $0.36(0.11-0.69)$ & $0.94(0.89-0.97)$ \\
\hline Sawit et al. [25] & 70 & 2 & 11 & 0 & 57 & $1.00(0.16-1.00)$ & $0.84(0.73-0.92)$ \\
\hline Hur et al. [26] & 83 & 13 & 13 & 0 & 57 & $1.00(0.75-1.00)$ & $0.81(0.70-0.90)$ \\
\hline
\end{tabular}

$N$-number of patients, $F N$ - false negative, $F P$-false positive, $T N$ - true negative, $T P$ - true positive, $C l$-confidence interval

sitivity and specificity. The area under the curve $( \pm \mathrm{SE})$ was $0.93( \pm 0.02)$, indicating very good diagnostic accuracy.

To determine the implications of our results for clinical use, we calculated the probability of $\mathrm{CT}$ detecting LA/LAA thrombus. Table IV shows the likelihood ratio profile of $\mathrm{CT}$ (positive likelihood ratio $=$ 6.24; negative likelihood ratio $=0.22$ ) primarily as a tool for ruling out thrombus.
To assess possible explanations for the heterogeneity, we performed stratified analysis to identify sources of heterogeneity among included studies. Table IV presents three factors that were found to be most strongly associated with the observed heterogeneity. The estimated DOR was nearly 6.3 times lower with a prospective study design than no or unclear study design. Studies with CT types that used a dual-source CT scanner produced diag- 
A

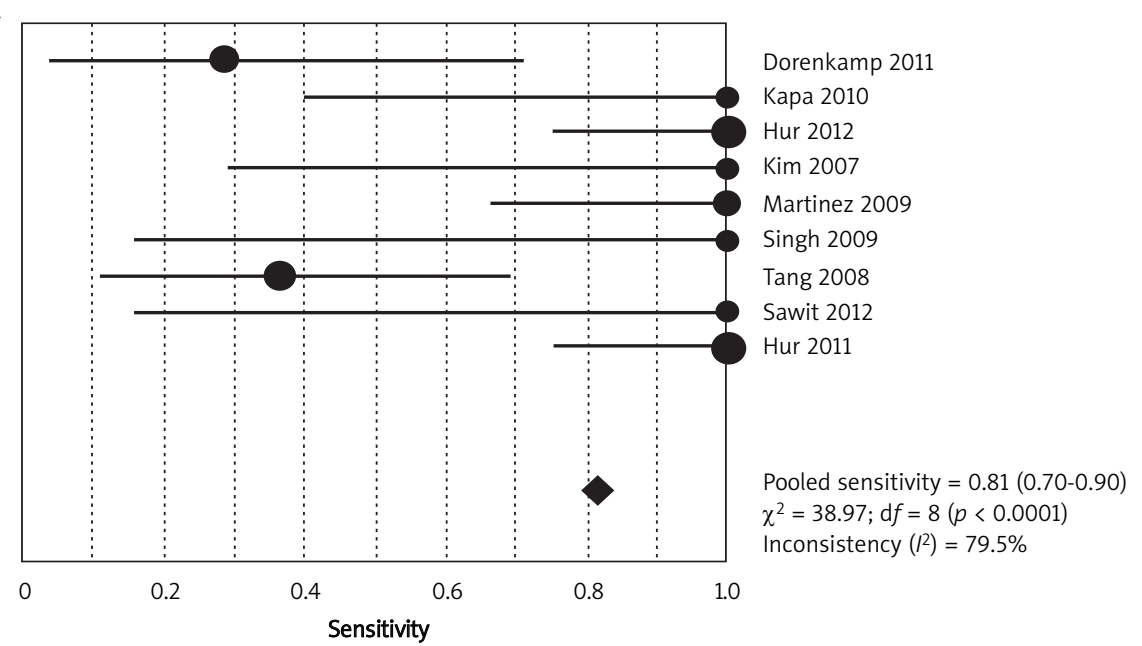

Sensitivity $(95 \% \mathrm{Cl})$

$0.29(0.04-0.71)$

$1.00(0.40-1.00)$

$1.00(0.75-1.00)$

$1.00(0.29-1.00)$

$1.00(0.66-1.00)$

$1.00(0.16-1.00)$

$0.36(0.11-0.69)$

$1.00(0.16-1.00)$

$1.00(0.75-1.00)$

B

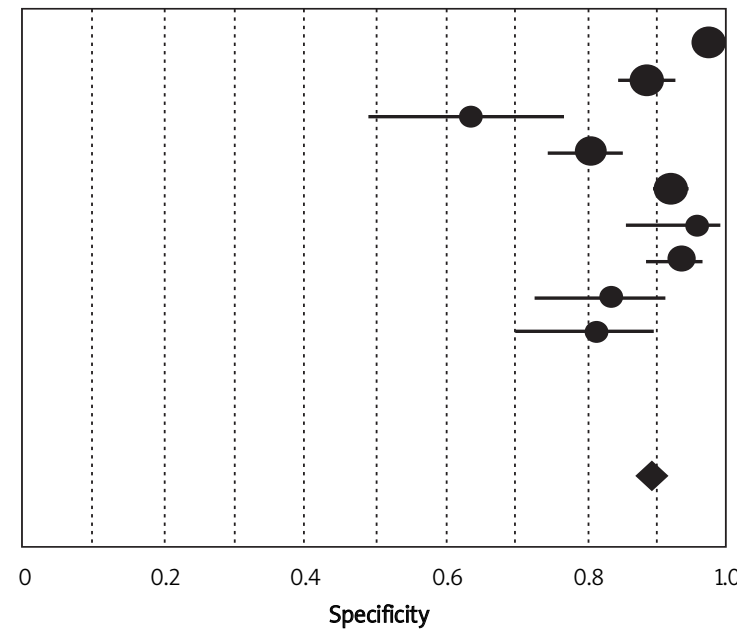

Dorenkamp 2011

Kapa 2010

Hur 2012

Kim 2007

Martinez 2009

Singh 2009

Tang 2008

Sawit 2012

Hur 2011

Specificity $(95 \% \mathrm{Cl})$

$0.98(0.95-0.99)$

$0.88(0.84-0.92)$

$0.64(0.49-0.77)$

$0.81(0.75-0.86)$

$0.92(0.89-0.95)$

$0.96(0.86-1.00)$

$0.94(0.89-0.97)$

$0.84(0.73-0.92)$

$0.81(0.70-0.90)$

Figure 3. Forest plot of pooled sensitivity and specificity of CT in diagnosis of LA/LAA thrombus

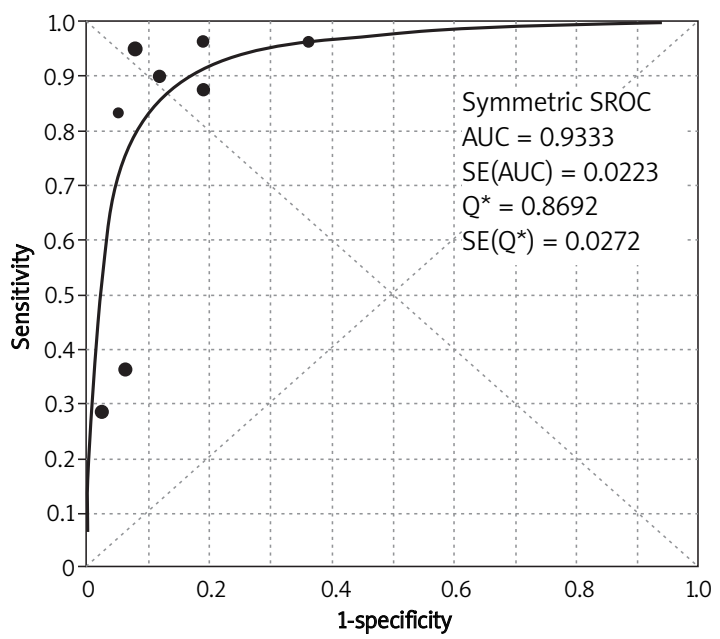

Figure 4. SROC for CT in diagnosis of LA/LAA thrombus $S R O C$ - summary receiver operating characteristic, $A \cup C$ - area under the curve nostic odds ratio estimates 2.3 times higher than studies that used other MDCT. In addition to the above two factors, quality of the study also affected the results. However, even after stratifying by study design and target sequence, considerable unexplained heterogeneity persisted in all the summary measures. The shape of the SROC curve sug gested that variability in diagnostic thresholds (cut-points) across studies could partly explain the heterogeneity.

The evaluation of publication bias showed that the Egger test was significant $(p=0.003)$. The funnel plot also showed marked asymmetry, with studies missing from the left, suggesting a publication bias (Figure 5).

\section{Discussion}

Atrial fibrillation is the most common cause of embolic sources from the heart, and AF-related strokes constitute about $60 \%$ of all cardioembolic 
Table IV. Stratified analysis for the evaluation of heterogeneity in studies

\begin{tabular}{|c|c|c|c|c|c|}
\hline Group or subgroup & $\begin{array}{c}\text { Pooled } \\
\text { sensitivity } \\
(95 \% \mathrm{Cl}), \%\end{array}$ & $\begin{array}{c}\text { Pooled } \\
\text { specificity } \\
(95 \% \mathrm{Cl}), \%\end{array}$ & $\begin{array}{c}\text { Positive } \\
\text { likelihood } \\
\text { ratio }(95 \% \mathrm{Cl})\end{array}$ & $\begin{array}{c}\text { Negative } \\
\text { likelihood } \\
\text { ratio }(95 \% \mathrm{Cl})\end{array}$ & $\begin{array}{c}\text { Pooled } \\
\text { diagnostic } \\
\text { odds ratio }(95 \% \mathrm{Cl})\end{array}$ \\
\hline All reports & $0.81(0.70-0.90)$ & $0.90(0.88-0.91)$ & $6.24(4.05-9.63)$ & $0.22(0.08-0.59)$ & 26.44 (11.97-58.41) \\
\hline \multicolumn{6}{|l|}{ CT type: } \\
\hline Dual-source & $1.00(0.88-1.00)$ & $0.84(0.80-0.87)$ & $4.65(2.39-9.07)$ & $0.07(0.01-0.31)$ & 71.83 (13.44-383.95) \\
\hline Others & $0.65(0.46-0.80)$ & $0.91(0.90-0.93)$ & $7.55(4.63-12.31)$ & $0.41(0.18-0.94)$ & $21.53(8.07-57.41)$ \\
\hline \multicolumn{6}{|l|}{ Study quality: } \\
\hline QUADAS item > 10 & $0.74(0.59-0.86)$ & $0.92(0.90-0.94)$ & $5.54(3.03-10.13)$ & $0.34(0.12-0.99)$ & $22.18(7.32-67.20)$ \\
\hline QUADAS item $\leq 10$ & $1.00(0.81-1.00)$ & $0.88(0.86-0.90)$ & $7.00(4.35-11.26)$ & $0.12(0.03-0.44)$ & $60.04(13.59-265.19)$ \\
\hline \multicolumn{6}{|c|}{ Prospective study design: } \\
\hline$\overline{Y e s}$ & $0.33(0.13-0.59)$ & $0.96(0.94-0.98)$ & $7.33(3.30-16.27)$ & $0.70(0.51-0.97)$ & $10.72(3.59-32.01)$ \\
\hline No or unclear & $1.00(0.92-1.00)$ & $0.87(0.85-0.89)$ & $6.04(3.69-9.90)$ & $0.10(0.04-0.27)$ & $67.50(21.85-208.48)$ \\
\hline
\end{tabular}

Cl - confidence interval, QUADAS - Quality Assessment of Diagnostic Accuracy Studies

strokes [4]. Prevalence of AF is a major risk factor not only for stroke, but also for other cardiovascular events, such as cardiovascular death, unstable angina and myocardial infarction. One report has shown that the annual incidence of nonfatal stroke and unstable angina was higher, and cardiovascular death was more than double, in AF versus nonAF patients. Although numerically higher with $A F$, there was little difference in the rate of nonfatal myocardial infarction between $A F$ and non-AF patients [27]. There were notable differences in the baseline characteristics and risk factor profile of patients with AF compared with non-AF patients, with older age, higher prevalence of hypertension, and a larger waist circumference. These differences may contribute to cardiovascular events for patients with AF [27].

Thrombi of the LA and LAA are common sources of stroke, and because LA and LAA thrombi are treatable sources of embolism, the detection of thrombi may significantly affect patient management. Currently, TEE is considered the reference standard for the detection of intracardiac thrombus. The main advantages of TEE include high temporal and spatial resolution, as well as the lack of radiation and contrast exposure. However, TEE requires special skills for proper performance and interpretation. Additionally, it is a semi-invasive test, usually performed under conscious sedation [9, 10, 28]. Multi-detector spiral CT allows visualization of the entire LA, including the LAA. In most cases, anatomic rendering of the left atrium and pulmonary veins using CT is performed in addition to TEE to exclude the presence of thrombus in the LAA. Contrast enhanced cardiac MDCT provides high-resolution volumetric data sets of the heart and adjacent anatomical structures. It would be a clinically useful setting to rule out LA/LAA thrombus with

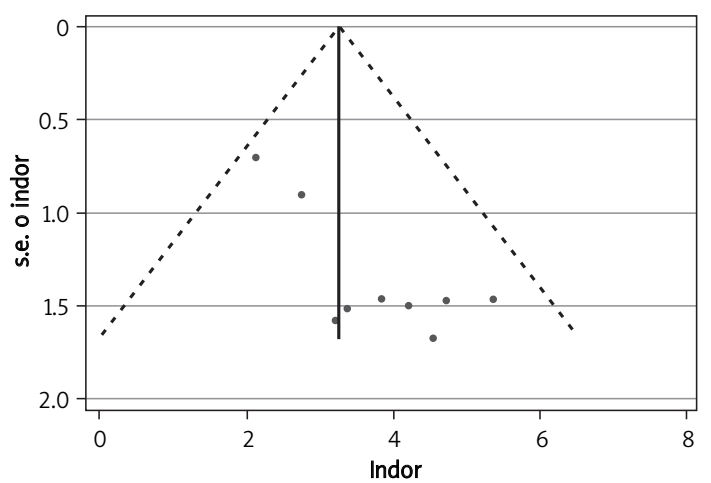

Figure 5. Funnel plot of this meta-analysis

the identical MDCT data set, obviating the need for pre-procedural TEE.

Alternative diagnostic approaches for the visualization of LA/LAA thrombus must prove their accuracy, reliability, and prognostic value before they can be recommended for clinical practice. We performed a meta-analysis of the diagnostic performance of CT as a potential noninvasive method for detecting LA/LAA thrombus compared with TEE as the reference standard. Table III displays the sensitivity and specificity estimates from each of the 9 studies in detecting LA/LAA thrombus. Both sensitivity (range: 0.29-1.0) and specificity (range: 0.640.98 ) estimates were highly variable. Among these reports, two prospective reports have very low sensitivities (29\% and $36 \%$, respectively) $[18,24]$ and others have $100 \%$ sensitivity. Differences in sensitivity and specificity between the studies may be presumed to be partially attributable to: (i) a relatively low rate of thrombus occurrence in the studied patient populations; (ii) contrast bolus techniques and (iii) different experience levels of the interpreting physicians. Despite the high hetero- 
geneity, we observed a good weighted sensitivity of $81 \%$ and specificity of $90 \%$ for CT in the detection of LA/LAA thrombus as compared with TEE. The area under the SROC curve $( \pm \mathrm{SE})$ was 0.93 ( \pm 0.02$)$, indicating very good diagnostic accuracy.

of note, we found significant heterogeneity in results among the included studies. Thus, our results should be interpreted cautiously, although our use of a random-effects model meta-analysis should correct for this issue at least in part. Use of stratified analysis may explain some of the sources of heterogeneity. Computed tomography type, study quality and prospective study design may be sources of the heterogeneity. Funnel plot of included studies showed a significant publication bias as well.

Intracavitary LA/LAA thrombi present as filling defects on MDCT. However, such defects may also result from inadequate mixing of contrast agent and blood and may even give the false-positive appearance of thrombus. Conditions associated with altered atrial blood-flow patterns, in particular AF, may favor the occurrence of filling artifacts that are incorrectly interpreted as thrombus [23, 24].

Our meta-analysis contains some limitations. First, most of the included studies performed the 2 examinations within a 3-day period but not on the same day. Thrombus could have formed or been lysed in the time between the TEE and CT examinations. Second, differences in the experience levels of the interpreting physicians could contribute to differences in results between studies. Third, only 2 of the studies had a prospective design; one limitation is the reliance on chart review for data collection. Fourth, because meta-analysis combines or integrates the results of several independent studies, the quality and reliability depend on the quality of included studies. We used the QUADAS tool for assessing methodological quality of individual studies as they were reported. Though 8 of the 9 studies fulfilled more than 10 items of the 14-item QUADAS checklist, the included studies we reviewed scored poorly on selection criteria, relevant clinical information and reference standard execution. The high number of items that scored "unclear" shows the need for further improvement in this regard. Finally, a common limitation in undertaking meta-analyses is the issue of publication bias, in which clinical trials with statistically significant results are published and those with undesirable results frequently are not [29]. The funnel plot of included studies suggests the presence of publication bias, which may have led to an overestimation of the true diagnostic performance. Other very different mechanisms can also lead to asymmetry in funnel plots, including true heterogeneity, data irregularities, artifacts and chance.
Transesophageal echocardiography is not only the imaging method of choice for the detection of LAA thrombus, but is also able to detect cardioembolic sources such as patent foramen ovale, valvular vegetations, and mobile thrombi in the aorta. Transesophageal echocardiography is also a valuable tool to image thoracic aortic atheroma associated with the prevalence of high-risk coronary anatomy [30]. In addition, in contrast to CT, TEE can be performed in patients with renal dysfunction or an allergy to contrast media. However, TEE is a time-consuming, invasive, and uncomfortable procedure. Furthermore, thrombus evaluation involves individual judgment that is reader dependent. Therefore, in clinical practice, a less invasive modality that is capable of assessing intracardiac thrombus in the setting of embolic stroke is desirable. With ongoing technical developments, CT might have more potential in detecting LA/LAA thrombus. Additional detector rows and improvements in temporal resolution will advance CT technology. Thus, we believe that CT can be used as an alternative modality for detecting thrombus in selected patients with stroke or AF, because it has high diagnostic accuracy for the detection of intracardiac thrombus, and is a noninvasive and reproducible modality. In addition, coronary artery calcium measurement, determined with $\mathrm{CT}$, is a powerful predictor of future cardiovascular risk [31].

Finally, we note that, even though CT is a noninvasive method compared to TEE, the radiation exposure is a disadvantage of CT. One report has mentioned that CT examinations may be associated with increased risk of future fatal cancer. This risk varies markedly and is considerably greater for women, younger patients, and for combined cardiac and aortic scans [32]. Hence further development of a system with a lower radiation exposure dose for use in clinical radiology will be necessary.

In conclusion, CT should be considered the foremost noninvasive alternative to TEE for detecting LA/LAA thrombus. Randomized studies at the patient level are needed to address the potential use of CT in detecting LA/LAA thrombus.

\section{References}

1. Bonita R. Epidemiology of stroke. Lancet 1992; 339: 342-4. 2. Pearson AC, Labovitz AJ, Tatineni S, et al. Superiority of transesophageal echocardiography in detecting cardiac source of embolism in patients with cerebral ischemia of uncertain etiology. J Am Coll Cardiol 1991; 17: 66-72.

3. Kapral MK, Silver FL. Preventive health care, 1999 update: 2. Echocardiography for the detection of a cardiac source of embolus in patients with stroke. Canadian Task Force on Preventive Health Care. CMAJ 1999; 161: 989-96.

4. Han SW, Nam HS, Kim SH, et al. Frequency and significance of cardiac sources of embolism in the TOAST classification. Cerebrovasc Dis 2007; 24: 463-8. 
5. Bernhardt P, Schmidt H, Hammerstingl C, et al. Patients with atrial fibrillation and dense spontaneous echo contrast at high risk a prospective and serial follow-up over 12 months with transesophageal echocardiography and cerebral magnetic resonance imaging. J Am Coll Cardiol 2005; 45: 1807-12.

6. Asinger RW, Koehler J, Pearce LA, et al. Pathophysiologic correlates of thromboembolism in nonvalvular atrial fibrillation: II. Dense spontaneous echocardiographic contrast (The Stroke Prevention in Atrial Fibrillation [SPAF-III] study). J Am Soc Echocardiogr 1999; 12: 1088-96.

7. Ellenbogen KA, Halperin JL, Le Heuzey JY, et al.; American College of Cardiology/American Heart Association Task Force on Practice Guidelines; European Society of Cardiology Committee for Practice Guidelines; European Heart Rhythm Association; Heart Rhythm Society. ACC/AHA/ESC 2006 Guidelines for the Management of Patients with Atrial Fibrillation: a report of the American College of Cardiology/American Heart Association Task Force on Practice Guidelines and the European Society of Cardiology Committee for Practice Guidelines (Writing Committee to Revise the 2001 Guidelines for the Management of Patients With Atrial Fibrillation): developed in collaboration with the European Heart Rhythm Association and the Heart Rhythm Society. Circulation 2006; 114: e257-354.

8. Fuster V, Rydén LE, Cannom DS, et al. HRS/EHRA/ECAS expert Consensus Statement on catheter and surgical ablation of atrial fibrillation: recommendations for personnel, policy, procedures and follow-up. A report of the Heart Rhythm Society (HRS) Task Force on catheter and surgical ablation of atrial fibrillation. Heart Rhythm 2007; 4: 816-61.

9. Omran H, Jung W, Rabahieh R, et al. Imaging of thrombi and assessment of left atrial appendage function: a prospective study comparing transthoracic and trans oesophageal echocardiography. Heart 1999; 81: 192-8.

10. Cujec B, Polasek P, Voll C, et al. Transesophageal echocardiography in the detection of potential cardiac source of embolism in stroke patients. Stroke 1991; 22: 727-33.

11. Moher D, Cook DJ, Eastwood S, et al. Improving the quality of reports of meta-analyses of randomised controlled trials: the QUOROM statement. Quality of Reporting of Metaanalyses. Lancet 1999; 354: 1896-900.

12. Whiting P, Rutjes AW, Reitsma JB, et al. The development of QUADAS: a tool for the quality assessment of studies of diagnostic accuracy included in systematic reviews. BMC Med Res Methodol 2003; 3: 25

13. Whiting PF, Weswood ME, Rutjes AW, et al. Evaluation of QUADAS, a tool for the quality assessment of diagnostic accuracy studies. BMC Med Res Methodol 2006; 6: 9.

14. Devillé WL, Buntinx F, Bouter LM, et al. Conducting systematic reviews of diagnostic studies: didactic guidelines. BMC Med Res Methodol 2002; 2: 9.

15. Deeks JJ. Systematic reviews in health care: Systematic reviews of evaluations of diagnostic and screening tests. BMJ 2001; 323: 157-62.

16. Higgins JP, Thompson SG, Deeks JJ, et al. Measuring inconsistency in meta-analyses. BMJ 2003; 327: 557-60.

17. Egger M, Davey Smith G, Schneider M, et al. Bias in metaanalysis detected by a simple, graphical test. BMJ 1997; 315: 629-34

18. Dorenkamp M, Sohns C, Vollmann D, et al. Detection of left atrial thrombus during routine diagnostic work-up prior to pulmonary vein isolation for atrial fibrillation: role of transesophageal echocardiography and multidetector computed tomography. Int J Cardiol 2011 Jul 15 [Epub ahead of print].
19. Kapa S, Martinez MW, Williamson EE, et al. ECG-gated dual-source CT for detection of left atrial appendage thrombus in patients undergoing catheter ablation for atrial fibrillation. J Interv Card Electrophysiol 2010; 29: 75-81.

20. Hur J, Kim YJ, Lee HJ, et al. Cardioembolic stroke: dualenergy cardiac CT for differentiation of left atrial appendage thrombus and circulatory stasis. Radiology 2012; 263: 688-95.

21. Kim Y, Klein AL, Halliburton SS, et al. Left atrial appendage filling defects identified by multidetector computed tomography in patients undergoing radiofrequency pulmonary vein antral isolation: a comparison with transesophageal echocardiography. Am Heart J 2007; 154 : 1199-205.

22. Martinez MW, Kirsch J, Williamson EE, et al. Utility of nongated multidetector computed tomography for detection of left atrial thrombus in patients undergoing catheter ablation of atrial fibrillation. JACC CardiovasC Imaging 2009; 2: 69-76.

23. Singh NK, Nallamothu N, Zuck VP, et al. Left atrial appendage filling defects on 64-slice multidetector computed tomography in patients undergoing pulmonary vein isolation: predictors and comparison to transesophageal echocardiography. J Comput Assist Tomogr 2009; 33: 946-51.

24. Tang RB, Dong JZ, Zhang ZQ, et al. Comparison of contrast enhanced 64-slice computed tomography and transesophageal echocardiography in detection of left atrial thrombus in patients with atrial fibrillation. J Interv Card Electrophysiol 2008; 22: 199-203.

25. Sawit ST, Garcia-Alvarez A, Suri B, et al. Usefulness of cardiac computed tomographic delayed contrast enhancement of the left atrial appendage before pulmonary vein ablation. Am J Cardiol 2012; 109: 677-84.

26. Hur J, Kim YJ, Lee HJ, et al. Dual-enhanced cardiac CT for detection of left atrial appendage thrombus in patients with stroke: a prospective comparison study with transesophageal echocardiography. Stroke 2011; 42: 2471-7.

27. Goto S, Bhatt DL, Röther J, et al. Prevalence, clinical profile, and cardiovascular outcomes of atrial fibrillation patients with atherothrombosis. Am Heart J 2008; 156: 855-63.

28. Wolber T, Maeder M, Atefy R, et al. Should routine echocardiography be performed in all patients with stroke? J Stroke Cerebrovasc Dis 2007; 16: 1-7.

29. Easterbrook PJ, Berlin JA, Gopalan R, et al. Publication bias in clinical research. Lancet 1991; 337: 867-72.

30. Shen X, Aronow WS, Nair CK, et al. Thoracic aortic atheroma severity predicts high-risk coronary anatomy in patients undergoing transesophageal echocardiography. Arch Med Sci 2011; 7: 61-6.

31. Kępka C, Pręgowski J, Michałowska I. Cardiac computed tomography. Clinical usefulness of calcium scoring and coronary computed tomography angiography. Postep Kardiol Inter 2010; 4: 178-86.

32. Einstein AJ, Henzlova MJ, Rajagopalan S. Estimating risk of cancer associated with radiation exposure from 64-slice computed tomography coronary angiography. JAMA 2007; 298: 317-23. 